\title{
OspC facilitates Borrelia burgdorferi invasion of Ixodes scapularis salivary glands
}

\author{
Utpal Pal, ${ }^{1}$ Xiaofeng Yang, ${ }^{2}$ Manchuan Chen, ${ }^{3}$ Linda K. Bockenstedt, ${ }^{1}$ John F. Anderson, ${ }^{4}$ \\ Richard A. Flavell, ${ }^{3}$ Michael V. Norgard, ${ }^{2}$ and Erol Fikrig ${ }^{1}$ \\ ${ }^{1}$ Section of Rheumatology, Department of Internal Medicine, Yale University School of Medicine, New Haven, \\ Connecticut, USA \\ ${ }^{2}$ Department of Microbiology, University of Texas Southwestern Medical Center, Dallas, Texas, USA \\ ${ }^{3}$ Section of Immunobiology, Yale University School of Medicine, New Haven, Connecticut, USA \\ ${ }^{4}$ Department of Entomology, Connecticut Agricultural Experiment Station, New Haven, Connecticut, USA
}

\begin{abstract}
Outer surface protein $\mathrm{C}(\mathrm{OspC})$ is a differentially expressed major surface lipoprotein of Borrelia burgdorferi. ospC is swiftly upregulated when spirochetes leave the Ixodes scapularis tick gut, migrate to the salivary gland, and exit the arthropod vector. Here we show that OspC strongly binds to the tick salivary gland, suggesting a role for $\mathrm{OspC}$ in spirochete adherence to this tissue. In vivo studies using a murine model of Lyme borreliosis showed that while $\mathrm{OspC} \mathrm{F}(\mathrm{ab})_{2}$ fragments did not influence either the viability of spirochetes or $o s p C$ gene expression, they did interfere with $B$. burgdorferi invasion of tick salivary glands. We then generated $o s p C$ knockout spirochetes in an infectious clone of B. burgdorferi and examined them within the vector. OspC-deficient or wild-type spirochetes persisted equally within the gut of unfed ticks and multiplied during the tick engorgement; however, unlike wild-type B. burgdorferi, the mutants were unable to invade salivary glands. Salivary gland colonization of OspC-deficient spirochetes was completely restored when this mutant was complemented in trans with a plasmid harboring the wild-type ospC gene. These studies conclusively demonstrate the importance of $\mathrm{OspC}$ in the invasion of tick salivary glands by B. burgdorferi, a critical step in the transmission of spirochetes from the arthropod vector to the mammalian host.
\end{abstract}

J. Clin. Invest. 113:220-230 (2004). doi:10.1172/JCI200419894.

\section{Introduction}

Borrelia burgdorferi is the causative agent of Lyme disease, a common arthropod-borne illness in the US, Europe, and parts of Asia $(1,2)$. The bacterium is maintained in an enzootic life cycle, which in the US mainly involves Ixodes scapularis ticks and Peromyscus leucopus, the whitefooted mouse (2). Larval ticks acquire spirochetes by feeding on infected mice and remain infested in the subsequent nymphal and adult stages of development. Ixodes ticks have the capacity to feed on a wide variety of vertebrate hosts and deposit spirochetes into the skin during engorgement (3). The bacterium soon spreads locally, often causing a skin rash known as erythema migrans in humans (4). After several weeks, the spirochetes may dis-

Received for publication August 27, 2003, and accepted in revised form November 4, 2003.

Address correspondence to: Erol Fikrig, Section of Rheumatology, Department of Internal Medicine, Yale University School of Medicine, New Haven, Connecticut 06520, USA. Phone: (203) 785-2453; Fax: (203) 785-7053;

E-mail: erol.fikrig@yale.edu.

Utpal Pal and Xiaofeng Yang contributed equally to this work.

Conflict of interest: The authors have declared that no conflict of interest exists.

Nonstandard abbreviations used: outer surface protein (Osp); salivary gland extract (SGE); $0.05 \%$ Tween-20 and $5 \%$ normal goat serum (PST); National Center for Biotechnology Information (NCBI); 6-carboxyfluorescein (FAM); 6-carboxy- $\mathrm{N}, \mathrm{N}, \mathrm{N}^{\prime}, \mathrm{N}^{\prime}$ tetramethylrhodamine (TAMRA); streptomycin resistance $\left.(\text { Strep })^{\mathrm{r}}\right)$; kanamycin resistance $\left(\operatorname{Kan}^{\mathrm{r}}\right)$. seminate to distant organs, including the joints, heart, and nervous system. Laboratory mice can be infected with $B$. burgdorferi and serve as an experimental model for the study of pathogen transmission (5).

B. burgdorferi has developed strategies to survive in diverse environments (6). Differential gene expression by the spirochete at specific locations within a host may contribute to this adaptive process. As examples, B. burgdorferi synthesizes a fibronectin-binding protein (BBK32), a decorin-binding protein (DbpA), a family of anticomplement proteins (Erp's), and several VlsE proteins in mammals, and these proteins facilitate spirochete dissemination and survival within the mammal (7-10). In contrast, outer surface protein A (OspA) and OspB are expressed mainly by B. burgdorferi in ticks (3). Spirochetes synthesize OspA when entering I. scapularis from an infected host and continue to produce abundant OspA within the resting tick (11). Recent studies have indicated that OspA mediates adherence of the spirochete to the tick gut (12). Taken together, these experiments support the notion that differentially expressed genes contribute to the B. burgdorferi survival in the reservoir host and the arthropod vector.

$\mathrm{OspC}$ is a major $23-\mathrm{kDa}$ protein expressed by $B$. burgdorferi during transmission of spirochetes from ticks to mammals as well as in the vertebrate host (13, 14). Interestingly, when an infested tick engorges, $B$. burgdorferi within the gut multiply and downregulate ospA (15). At the same time, the spirochetes start pro- 
ducing $\mathrm{OspC}$ in the feeding gut and continue to produce OspC throughout the transmission process and during the establishment of early vertebrate infection $(11,16)$. This pattern of expression suggests that OspC may serve a function in the tick, possibly facilitating the migration of the spirochete from the vector gut to the salivary glands during transmission (17). After transmission from the tick, OspC may also play a role in colonization of host tissues (18). Here, we have investigated the potential role of OspC as a spirochete ligand that binds to tick salivary glands and whether interference with OspC-vector interactions can prevent the invasion of I. scapularis salivary glands by B. burgdorferi in vivo.

\section{Methods}

B. burgdorferi and I. scapularis. A low-passage clonal isolate of B. burgdorferi $\mathrm{N} 40$ that is infectious for mice was used throughout the study, except for the generation of OspC-deficient $B$. burgdorferi, which were derived from a clonal, low-passage, virulent B. burgdorferi 297. Adult female $I$. scapularis ticks were collected in Connecticut. The egg masses were laid in the laboratory. Hatched larvae were allowed to feed on uninfected $\mathrm{C} 3 \mathrm{H} / \mathrm{HeN}$ mice to produce pathogen-free nymphs. The Yale University Institutional Animal Care and Use Committee approved experimental procedures involving the use of animals. ELISA and confocal microscopy to assess protein binding to the tick salivary glands. Recombinant OspC and ErpT (a representative control protein) from $B$. burgdorferi $\mathrm{N} 40$ were expressed and purified in their nonlipidated forms as previously described (12). OspC, ErpT, and BSA were labeled with fluorescein isothiocyanate (FITC) from Molecular Probes (Eugene, Oregon, USA). In a typical labeling reaction, $1 \mathrm{pmol}$ of OspC, ErpT, or BSA bound to 2.3, 2.1 , or $6.6 \mathrm{pmol}$ of FITC, respectively. Salivary glands from flat nymphal I. scapularis were dissected and homogenized in PBS on ice with a Kontes microhomogenizer (VWR, West Chester, Pennsylvania, USA) as described (12). Homogenized proteins from salivary glands isolated from one nymph or $100 \mu \mathrm{l}$ of soluble salivary gland extract (SGE) $(5 \mu \mathrm{g} / \mathrm{ml})$ or fetal bovine serum (FBS) (10 $\mu \mathrm{g} / \mathrm{ml}$ ) were used to coat microtiter wells (ICN, Costa Mesa, California, USA) and were incubated overnight at $4^{\circ} \mathrm{C}$. Nonspecific sites were blocked by incubation of SGE-coated wells with $15 \%$ FCS for 2 hours at $37^{\circ} \mathrm{C}$. Plates were then incubated with $100 \mu \mathrm{l}$ of FITC-labeled OspC, ErpT, or BSA $(10 \mu \mathrm{g} / \mathrm{ml})$ at $37^{\circ} \mathrm{C}$ for 1 hour. The samples were washed three times with PBS with $0.05 \%$ Tween-20 (PBS-Tween-20). Binding was detected using anti-FITC HRP-conjugated IgG (Amersham, Piscataway, New Jersey, USA) as a secondary reagent and a microwell peroxidase substrate (KPL, Gaithersburg, Maryland, USA) was used for color development. The OD was measured at $450 \mathrm{~nm}$ after 15 minutes.

Assessment of protein binding by confocal microscopy was performed as described (12). Salivary glands were dissected, placed on silylated glass slides (PGC, Gaithersburg, Maryland, USA), washed twice with PBS, and incubated with PBS-Tween-20 with 5\% FCS for 30 minutes at room temperature. Samples were then incubated for 1 hour at room temperature with FITC-labeled OspC, ErpT, or BSA $(50 \mu \mathrm{l}$ of $10 \mu \mathrm{g} / \mathrm{ml}$ of FITC-labeled protein). Samples were subsequently stained with propidium iodide (50 $\mu \mathrm{l}$ of a $10-\mu \mathrm{g} / \mathrm{ml}$ solution) for 3 minutes at room temperature, washed three times with PBS-Tween-20, and mounted in antifade reagent (Molecular Probes) for examination. The tissues were viewed using a Zeiss LSM 510 scanning laser confocal microscope equipped with an argon/krypton laser.

Antibodies and the generation and characterization of $F(a b)_{2}$ fragments. Generation of OspC polyclonal $\mathrm{Ab}$ against $B$. burgdorferi $\mathrm{N} 40$ has been described (19). A volume of 10 $\mathrm{ml}$ of normal rabbit sera or polyclonal antisera was passed over a $0.5-\mathrm{ml}$ protein A column (Bio-Rad, Hercules, California, USA), and the column was washed twice with $20 \mathrm{ml}$ of PBS, $\mathrm{pH}$ 7.4. The bound IgG was eluted in $1 \mathrm{ml}$ of $0.1 \mathrm{M}$ glycine, $\mathrm{pH} 3.0$, concentrated, and desalted with a spin column (Millipore, Bedford, Massachusetts, USA). Immobilized pepsin was used to generate $\mathrm{F}(\mathrm{ab})_{2}$ fragments from the whole IgG fraction of normal rabbit sera or OspC antisera [ImmunoPure $\mathrm{F}(\mathrm{ab})_{2}$ preparation kit; Pierce, Rockford, Illinois, USA]. Purified IgG was dialyzed overnight against $20 \mathrm{mM}$ sodium acetate buffer at $\mathrm{pH} 4.5$ and was concentrated to $20 \mathrm{mg} / \mathrm{ml} \mathrm{IgG}$. The IgG was added to a tube containing immobilized pepsin $(250$ $\mu \mathrm{g} / 10 \mathrm{mg} \operatorname{IgG}$ ) and was incubated for 4 hours at $37^{\circ} \mathrm{C}$. The cleaved $\mathrm{F}(\mathrm{ab})_{2}$ fragments were separated from $\mathrm{Fc}$ fragments or undigested IgGs using an immobilized protein A column. The eluate was dialyzed overnight against PBS, pH 7.4, within a tube with a molecular weight cutoff of $50 \mathrm{kDa}$ and was concentrated using spin columns. An aliquot of the $\mathrm{F}(\mathrm{ab})_{2}$ fragments was separated by $12 \%$ SDS-PAGE which demonstrated nearly $100 \%$ purity of the $\mathrm{F}(\mathrm{ab})_{2}$ fragments without any detectable IgGs or other contaminants.

The binding of $\mathrm{OspC} \mathrm{F}(\mathrm{ab})_{2}$ to B. burgdorferi lysates or whole spirochetes was analyzed by ELISA or immunofluorescence microscopy, respectively. For ELISA, soluble B. burgdorferi $\mathrm{N} 40$ proteins $(10 \mu \mathrm{g} / \mathrm{ml})$ were coated onto microtiter wells. Nonspecific sites were blocked with $15 \%$ FBS and samples were incubated with a 1:100 dilution of normal rabbit sera or OspC antiserum or $8 \mu \mathrm{g} / \mathrm{ml}$ of purified $\operatorname{IgGs}$ or $\mathrm{F}(\mathrm{ab})_{2}$. Bound antibodies were detected using an anti-rabbit $\mathrm{F}(\mathrm{ab})_{2}$ fragment-specific goat IgG conjugated to HRP (Jackson Immunoresearch, West Grove, Pennsylvania, USA). In selected wells, primary Ab was omitted to measure nonspecific binding. Binding of $\mathrm{OspCF}(\mathrm{ab})_{2}$ fragments to the surface of $B$. burgdorferi was performed as described (20) with the following modifications. A volume of $5 \mu \mathrm{l}$ of spirochetes $\left(10^{9} / \mathrm{ml}\right)$ in PBS was spotted on silylated glass slides, allowed to dry on slides, and incubated for 30 minutes in PBS supplemented with $0.05 \%$ Tween- 20 and $5 \%$ normal goat serum (PST) at room temperature. Samples were then incubated with $\mathrm{F}(\mathrm{ab})_{2}$ fragments $(10-100 \mu \mathrm{g} / \mathrm{ml})$ prepared either from normal rabbit sera or OspC antisera in PST at room temperature for 1 hour. Samples were washed twice for 5 minutes in PBS and incubated with an anti-rabbit $\mathrm{F}(\mathrm{ab})_{2}$ 
fragment specific goat IgG (1:100 dilution) conjugated to FITC (Jackson Immunoresearch) at room temperature for 1 hour. The samples were washed three times with PBS and mounted in antifade reagent. The spirochetes were imaged by the Zeiss LSM 510 confocal microscope. Bactericidal assay. $\mathrm{F}(\mathrm{ab})_{2}$ fragments were tested for their bactericidal activity against $B$. burgdorferi $\mathrm{N} 40$ by dark-field microscopy as described (20). Briefly, spirochetes $\left(5 \times 10^{6} / \mathrm{ml}\right)$ were incubated in BSK-H medium (Sigma-Aldrich, St. Louis, Missouri, USA) supplemented with purified $\mathrm{F}(\mathrm{ab})_{2}$ fragments (50 $\mu \mathrm{g} / \mathrm{ml}$ ) for $24-48$ hours at $33^{\circ} \mathrm{C}$. Anti-B. burgdorferi serum from a patient with late-stage Lyme disease served as a control in the bactericidal assay. The percentage of viable spirochetes was determined by darkfield microscopic observation of the loss of spirochete motility and refractivity in ten random fields by experimenters "double-blinded" to sample identity. In addition, $50-\mu 1$ aliquots from each control or $\mathrm{F}(\mathrm{ab})_{2}$-treated groups were removed, incubated with $500 \mu \mathrm{l}$ of BSK-H medium at $33^{\circ} \mathrm{C}$ for 5 days, and examined for viable spirochetes.

In vivo invasion studies. Pathogen-free $\mathrm{NCr}$ mice were purchased from the NIH. Spirochete-infested nymphs were generated from I. scapularis larva that fed on $B$. burgdorferi $\mathrm{N} 40$-infected $\mathrm{C} 3 \mathrm{H} / \mathrm{HeN}$ mice and molted into nymphs. The animals (three mice per group) were injected (100 $\mu \mathrm{l}$ intraperitoneally and $100 \mu \mathrm{l}$ subcutaneously) with selected $\mathrm{F}(\mathrm{ab})_{2}$ fragments (100 $\mu \mathrm{g} /$ mouse). Twenty-four hours later, 25 infected $I$. scapularis nymphs were placed on each mouse. The animals were again treated with $\mathrm{F}(\mathrm{ab})_{2}$ fragments on the next day to maintain an effective concentration of $\mathrm{F}(\mathrm{ab})_{2}$ in the blood. Batches of feeding nymphs were removed from the mouse skin at 24-hour intervals after attachment. Remaining nymphs fed to repletion and detached from the mice, usually at 72-96 hours. Salivary glands and guts from each group of nymphs were dissected under a microscope in sterile PBS and examined by RT-PCR and confocal microscopy at 24, 48, 72, and 96 hours after tick attachment.

Organs from nymphal ticks were prepared for microscopy as described previously (20). Salivary glands or gut were dissected, washed three times in PBS, placed on silylated glass slides, allowed to dry, and fixed with acetone for 5 minutes. Fixed slides were rinsed twice with PBS and incubated for 30 minutes with PST at room temperature. Organs were incubated with an affinity-purified FITC-labeled goat anti-B. burgdorferi (KPL) at a dilution of 1:50 in PST at room temperature for 1 hour. The samples were counterstained with propidium iodide ( $50 \mu \mathrm{l}$ of a $10-\mu \mathrm{g} / \mathrm{ml}$ solution) and viewed with the Zeiss LSM 510 confocal microscope. The distribution of spirochetes inside the salivary glands and gut was determined by scanning of the entire organ from end to end on the horizontal axis and throughout its depth on the vertical plane at each point.

RT-PCR and quantitative PCR. Oligonucleotide primers used for RT-PCR and quantitative PCR studies are described in Supplementary Table 1, http://www.jci. org/cgi/content/full/113/2/220/DC1. As an internal control for the loading of cDNA isolated from infected ticks, a portion of the I. scapularis $\beta$-actin gene was amplified and cloned by PCR. Degenerate primers were synthesized based on known tsetse fly $\beta$-actin gene sequence (21), and PCR was performed with I. scapularis cDNA as template. The amplified I. scapularis $\beta$-actin gene product was cloned into a TA vector, PCR 2.1 (Invitrogen, Carlsbad, California, USA), which had over $90 \%$ sequence homology to known arthropod $\beta$-actin genes in GenBank (National Center for Biotechnology Information). A set of $I$. scapularis $\beta$-actin specific forward and reverse primers were designed and used for expression studies. The primer pair produced a single 400-bp amplicon from I. scapularis DNA template but did not generate a product when murine or spirochete DNA was used as template (data not shown). The $I$. scapularis $\beta$-actin sequence data were submitted to NCBI GenBank with the accession number AF426178.

RT-PCR detection of B. burgdorferi was performed as described (22). Fed I. scapularis were removed from the mice at specific time intervals and total RNA was isolated from gut or salivary glands using the NucleoSpin RNA II Kit (Clontech, Palo Alto, California, USA). Total RNA $(2 \mu \mathrm{g})$ was converted to cDNA using the ProSTAR First Strand RT-PCR Kit (Stratagene, La Jolla, California, USA) with random hexamers and was used for PCR to amplify 333-bp B. burgdorferi N40 flagellin $(f l a B)$ transcripts. In selected tubes, RT-PCR was conducted without the addition of reverse transcriptase to confirm the absence of DNA contamination. An aliquot of the RT-PCR product was analyzed on a $1.5 \%$ agarose gel.

For quantitative analysis of the flaB or $\operatorname{sp} p C$ transcripts by real-time PCR, total RNA was isolated from the guts of fed I. scapularis nymphs. RNA $(2 \mu \mathrm{g})$ was converted to CDNA, and quantitative PCR was performed on a Bio-Rad iCycler according to the manufacturer's instructions. The oligonucleotide probes for real-time PCR were synthesized by ABI (Applied Biosystems, Foster City, California, USA) and contained a $5^{\prime}$ reporter, 6-carboxyfluorescein (FAM), and a $3^{\prime}$ quencher, 6-carboxy- $\mathrm{N}, \mathrm{N}, \mathrm{N}^{\prime}, \mathrm{N}^{\prime}$-tetramethylrhodamine (TAMRA). The 70-bp B. burgdorferi $\mathrm{N} 40$ flaB and 128-bp ospC and a 122-bp fragment of the I. scapularis $\beta$-actin gene were amplified using the following cycles: $95^{\circ} \mathrm{C}$ for $4 \mathrm{~min}$ utes, and 40 cycles of $95^{\circ} \mathrm{C}$ for 15 seconds and $60^{\circ} \mathrm{C}$ for 1 minute. Standard curves for flaB, ospC, or $\beta$-actin were prepared using tenfold serial dilutions of known quantities (10 to $10^{-7} \mathrm{ng}$ ) of B. burgdorferi DNA or the plasmid PCR 2.1- $\beta$-actin. The amount of tick cDNA in each sample was first normalized by measurement of levels of tick $\beta$-actin transcripts in quantitative PCR reaction, and equal amounts of tick CDNA were then used for measuring levels of $B$. burgdorferi fla $B$ or osp $C$ transcripts. For quantitative analysis of the B. burgdorferi burden in mice, DNA was isolated from murine skin samples using the DNeasy Tissue Kit (Qiagen Inc., 
Valencia, California, USA). Real-time PCR was then performed on equal amounts of isolated DNA using flaB primers as described above.

Generation of OspC-deficient and OspC-complemented B. burgdorferi. A clonal isolate of infectious B. burgdorferi 297 was used to generate an OspC-deficient mutant because of previous success with genetic manipulation of strain 297 (23). B. burgdorferi N40 and B. burgdorferi 297 are both prototypic $B$. burgdorferi sensu stricto strains with a high degree of OspC homology (24). Primers used to generate the OspC-deficient $B$. burgdorferi are indicated in Supplementary Table 2, http://www.jci.org/cgi/ content/full/113/2/220/DC1. The origin of the resistance markers used for the genetic manipulation of $B$. burgdorferi 297 in this study has been published $(25,26)$. Two $1.75-\mathrm{kb}$ DNA fragments were amplified by PCR from B. burgdorferi 297 genomic DNA using either primer pair 1 and 7 (to amplify the DNA fragment containing sequence upstream of the $\operatorname{cs} p C$ ) or primers 2 and 8 (to generate the DNA fragment containing part of the ospC open reading frame as well as downstream sequence of $\operatorname{sspC}$ ). A BglII site was engineered at the $5^{\prime}$ end of primers 7 and 8 . The resulting two PCR fragments were digested with $B g l \mathrm{II}$ and ligated together. A $3.5-\mathrm{kb}$ fragment was then amplified by PCR using primers 1 and 2 and was subsequently cloned into the PCR-XL-TOPO cloning vector (Invitrogen). The resulting plasmid was designated pOspC-BglII. Then, an aadA cassette (which confers streptomycin resistance [Strep $\mathrm{p}^{\mathrm{r}}$ to B. burgdorferi) driven by a flgB promoter from B. burgdorferi (26) was inserted at the BglII site of pOspC-BgIII, resulting in plasmid pOspC-Strep. For generation of the OspC mutant, $20 \mu \mathrm{g}$ of pOspC-Strep plasmid DNA was electroporated into an infectious clone of B. burgdorferi 297, designated $\mathrm{BbAH} 130$, as previously described (23). After the samples recovered overnight, the transformation mixture was then plated on pBSK agar medium containing $50 \mu \mathrm{g} / \mathrm{ml}$ of streptomycin (Sigma-Aldrich). More than 50 recombinants with Strep ${ }^{r}$ were recovered within 2 weeks of plating. PCR amplification with various primer pairs was performed on whole-cell lysates of these transformants to verify that transformants with Strep ${ }^{r}$ contained the correct aadA insertion. Allelic exchange was selected by double cross-over. PCR analysis for the presence of all known 21 endogenous plasmids in strain 297 was also performed as described previously (27).

To complement the OspC-deficient B. burgdorferi with a wild-type ospC gene, shuttle vector pBSV2-ospC (28), which confers kanamycin resistance $\left(\operatorname{Kan}^{\mathrm{r}}\right)(25)$, was constructed. First, a 1-kb DNA fragment containing a wildtype ospC gene was amplified by PCR from $B$. burgdorferi 297 using primers 9 and 10 (Supplementary Table 2, http://www.jci.org/cgi/content/full/113/2/220/DC1) and was subsequently cloned into PCR-XL-TOPO (Invitrogen), resulting in plasmid pXT-ospC. pXT-ospC was then digested with HindIII and $\mathrm{XbaI}$, and the 1-kb fragment containing the ospC gene was inserted into the corresponding sites of shuttle vector pBSV2, kindly provided by Philip Stewart (Rocky Mountain Laboratories, National
Institute of Allergy and Infectious Diseases, NIH, Hamilton, Montana, USA) (28). The resulting plasmid was designated pBSV2-ospC. For complementation, $20 \mu \mathrm{g}$ of pBSV2-ospC DNA was electroporated into the OspC-deficient B. burgdorferi, and about 20 clones with $\mathrm{Kan}^{\mathrm{r}} / \mathrm{Strep}^{\mathrm{r}}$ were obtained. At the concentrations of $200 \mu \mathrm{g} / \mathrm{ml}$ kanamycin and $50 \mu \mathrm{g} / \mathrm{ml}$ of streptomycin used in the present study, we did not observed any cross-resistance between these two antibiotic markers. To confirm that the complemented clones contained the pBSV2-ospC plasmid, whole-cell lysates from $10^{7} \mathrm{~B}$. burgdorferi cells with $\mathrm{Kan}^{\mathrm{r}} / \mathrm{Strep}^{\mathrm{r}}$ were used to transform TOP10 Escherichia coli chemical-competent cells (Invitrogen). More than 100 $\mathrm{Kan}^{\mathrm{r}} / \mathrm{Strep}^{\mathrm{r}}$-positive colonies/plate were obtained. Plasmids were rescued from five randomly selected $E$. coli transformants, and restriction digestion was performed to verify the recovery of plasmid pBSV2-ospC.

Microinjection of B. burgdorferi into ticks. For the generation of wild-type or OspC-deficient B. burgdorferi-infected ticks, a microinjection method was developed to introduce live spirochetes into the guts of I. scapularis nymphs. For microinjection, cells were grown in BSK-H medium (Sigma-Aldrich) and concentrated to a density of $1 \times 10^{9}$ spirochetes $/ \mathrm{ml}$. Then, $10 \mu \mathrm{l}$ of the cells was loaded into a glass capillary needle $1 \mathrm{~mm}$ in diameter (World Precision Instruments, Sarasota, Florida, USA) using a microloader (Eppendorf, Hamburg, Germany). Unfed I. scapularis nymphs were attached with the ventral side facing up onto a glass slide using double-sided tape. The tip of the capillary needle with the spirochete solution was carefully inserted into the rectal aperture through the forced opening of the rectal valves and the solution was injected with the aid of a stereo dissecting binocular microscope (Olympus, Melville, New York, USA) and a femtojet microinjector system (Eppendorf). The microinjection setup included an injection pressure of $1,000 \mathrm{hPa}$, an injection time of 1 second, and a compensation pressure of $8 \mathrm{hPa}$. The enema technique produced no mortality in injected nymphs. After microinjection, ticks were reared in a humid chamber for several days. Groups of ticks were sacrificed and assessed for spirochetes by dark-field microscopy, culture, and RT-PCR. Spirochetes $\left(10^{3}\right)$ were injected into ticks, and groups of ticks with spirochete infestation rates of approximately $100 \%$ were then used for the experimental in vivo invasion studies.

Statistical analysis. Results are expressed as the mean \pm SEM. The significance of the difference between the mean values of the groups was evaluated using the Student's $t$-test with StatView software (SAS Institute, Cary, North Carolina, USA).

\section{Results}

OspC binds to the I. scapularis salivary gland. When an infected tick takes a blood meal, B. burgdorferi within the gut produces OspC and invades tick salivary glands before migrating to the vertebrate host. We have previously shown that OspC does not significantly bind to the $I$. scapularis gut (12). To explore the possibility that OspC interacts with the tick salivary gland, we examined the 
Table 1

Borreliacidal activity of $\mathrm{OspC} F(\mathrm{ab})_{2}$ fragments against $B$. burgdorferi

\begin{tabular}{lcc}
\hline & \multicolumn{2}{c}{ Viable B. burgdorferi } \\
& $24 \mathrm{~h}$ & $48 \mathrm{~h}$ \\
Control (no addition) & 100 & 100 \\
$\mathrm{NRS} \mathrm{F}(\mathrm{ab})_{2}$ & $102 \pm 2.5$ & $96 \pm 13$ \\
OspC F $(\mathrm{ab})_{2}$ & $106 \pm 4$ & $119 \pm 12.5$ \\
B. burgdorferi antisera & $11 \pm 9$ & 0
\end{tabular}

B. burgdorferi $\mathrm{N} 40$ were incubated in BSK-H medium in the absence (Control) or presence of $\mathrm{F}(\mathrm{ab})_{2}$ fragments prepared from normal rabbit sera [NRS $\left.\mathrm{F}(\mathrm{ab})_{2}\right]$, polyclonal anti-OspC sera $\left[\mathrm{OspC} F(a b)_{2}\right]$, or a borreliacidal serum from a patient with diagnosed Lyme disease. The number of spirochetes was assessed by dark-field microscopy after 24 and 48 hours, and data are expressed relative to controls without $\mathrm{Ab}$ treatment. Data represent the number of spirochetes remaining viable after the treatment (mean $\pm \mathrm{SEM}, n=3$ ). Differences between control and NRS F $(a b)_{2}-$ as well as OspC F $(a b)_{2}$-treated samples were not statistically significant (Student's $t$-test).

adherence of OspC to soluble tick SGE by ELISA. Binding of recombinant FITC-labeled OspC from B. burgdorferi $\mathrm{N} 40$, a prototypic infectious $B$. burgdorferi sensu stricto isolate, was assessed. OspC strongly bound to SGE compared with other control proteins, such as BSA or B. burgdorferi ErpT, which adhered poorly to the SGE $(P<0.001)$ (Figure 1a). The binding was specific, as OspC bound only weakly to antigens in FBS (Figure 1a). OspC also adhered strongly to unfixed tick salivary gland when examined by confocal microscopy (Figure 1b). Compared with FITC-labeled OspC, the control proteins FITC-labeled ErpT and FITC-labeled BSA bound poorly to the tick salivary gland (Figure $1 \mathrm{~b}$ ).

$\mathrm{OspCF}(a b)_{2}$ fragments reduce the invasion of salivary glands by B. burgdorferi. Since OspC binds to the tick salivary gland, we assessed whether $B$. burgdorferi coated with OspC Ab could effectively enter and colonize this organ. Bactericidal effects of $B$. burgdorferi antibodies within ticks have been reported $(15,29,30)$. We therefore generated $\operatorname{IgG~} \mathrm{F}(\mathrm{ab})_{2}$ fragments from polyclonal OspC antisera so that interference with spirochete invasion of the gland could be distinguished from the borreliacidal effect of OspC antibodies. ELISA with B. burgdorferi lysates and immunofluorescence studies using unfixed spirochetes showed specific and comparable binding of $\mathrm{F}(\mathrm{ab})_{2}$ fragments and OspC antisera to the surface of $B$. burgdorferi (Figure 2); however, no significant bactericidal activity was found when B. burgdorferi were exposed to OspC $\mathrm{F}(\mathrm{ab})_{2}$ fragments in vitro (Table 1).

For in vivo invasion studies, mice were injected with $\mathrm{F}(\mathrm{ab})_{2}$ fragments prepared from either normal rabbit IgG or anti-OspC IgG before B. burgdorferi-infested nymphs were placed on the mice. The numbers of spirochetes inside the salivary glands or gut at different time points after tick attachment was assessed using confocal microscopy. SCID mice were used in these studies, as the effects of the administered $\mathrm{F}(\mathrm{ab})_{2}$ fragments could then be evaluated without the confounding influence of host antibodies on the spirochete. When guts of feeding ticks were stained for B. burgdorferi at 24-96 hours after the onset of feeding, comparable numbers of spirochetes were noted within the ticks that fed on the immunized mice, indicating that $\mathrm{OspC} \mathrm{F}(\mathrm{ab})_{2}$ fragments did not interfere with the multiplication or survivability of the spirochetes within the tick gut (Figure 3a, bottom row). In ticks that fed on mice administered normal rabbit IgG $\mathrm{F}(\mathrm{ab})_{2}$ fragments, spirochetes could be found within the salivary glands between 48 and 96 hours after the onset of feeding (Table 2 and Figure 3a, top left panel). In contrast, invasion of salivary glands was significantly blocked in ticks that fed on mice receiving OspC $\mathrm{F}(\mathrm{ab})_{2}$ (Table 2, $P<0.04$ at all the time point assessed, and Figure 3a, top right panel). Detection of viable spirochetes within the feeding gut or colonized salivary glands was further evaluated by RT-PCR analyses of fla $B$ transcripts. At 48 and 96 hours of feeding, comparable levels of fla $B$ RNA could be detected in the tick gut (Figure $3 b$, right panels). In contrast, lower levels of flaB RNA were evident in the salivary glands of ticks treated with $\mathrm{OspC} \mathrm{F}(\mathrm{ab})_{2}$ than in those of controls (Figure 3b, left panels). These data indicate that OspC plays an important role in the invasion of B. burgdorferi into the tick salivary glands during transmission from the vector to the murine host.

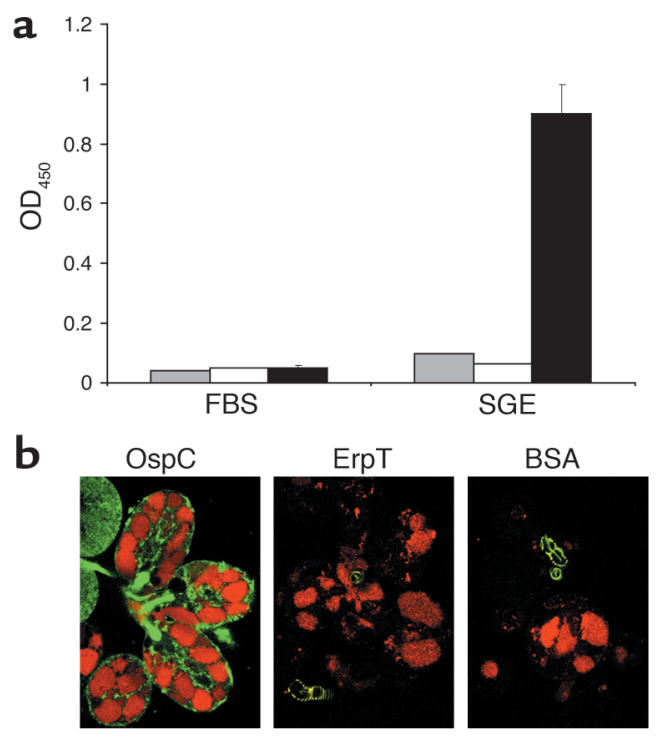

\section{Figure 1}

OspC binds to I. scapularis salivary glands. (a) FITC-labeled OspC (black bars), ErpT from B. burgdorferi N40 (white bars), and BSA (gray bars) were used to probe SGE- or FBS-coated wells. Data represent the $\mathrm{OD}_{450}$ at 15 minutes (mean $\pm \mathrm{SEM}, n=3$ ). The differences between the binding of OspC to SGE and its binding to BSA or ErpT are highly significant $(P<0.001)$. (b) Direct binding of FITC-labeled OspC to the intact unfixed tick salivary gland was detected using confocal microscopy. FITC-labeled ErpT and FITC-labeled BSA were used as controls. After the tick salivary gland was probed with FITClabeled protein (shown in green), the tissues were stained with propidium iodide to localize the nuclei of the salivary gland cells (shown in red). The objects stained green in the ErpT and BSA panels are not a part of the salivary gland. The endogenous weak green fluorescence of tick salivary glands was adjusted in the confocal microscope to compare the binding of different FITC-labeled antigens. The FITC and propidium iodide images were examined at $\times 400$ magnification and are presented as merged images for clarity $(n=3)$. 


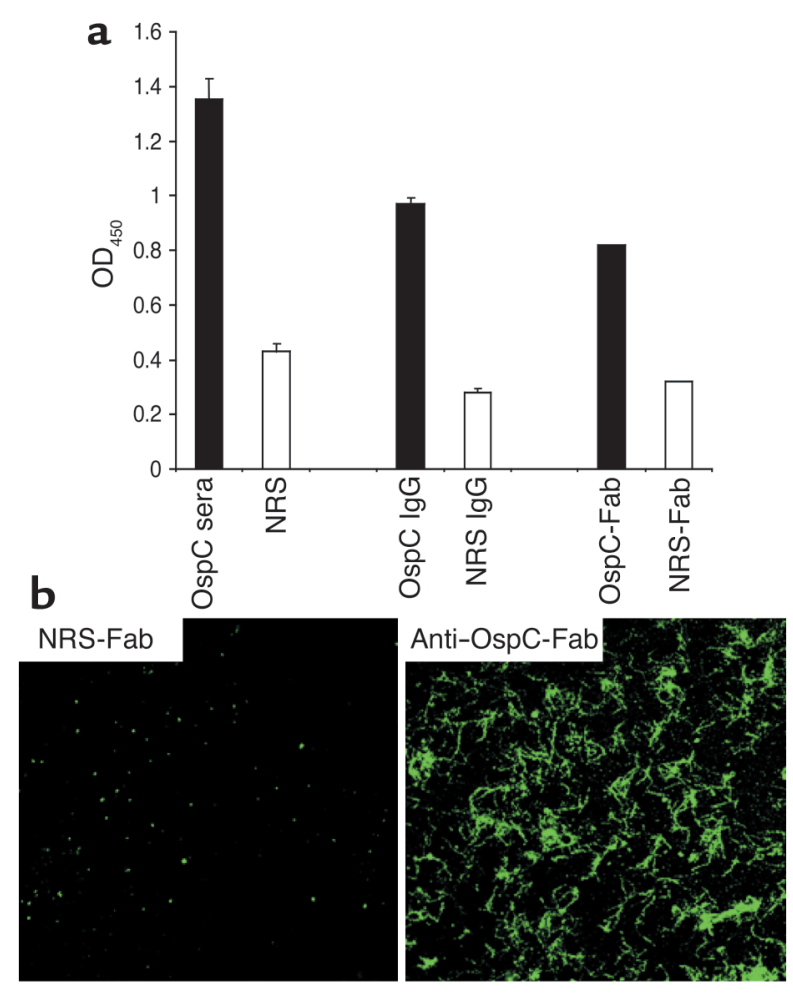

$\mathrm{OspCF}(a b)_{2}$ did not influence the number of spirochetes or ospC expression by B. burgdorferi in feeding gut. Previous studies have suggested that $B$. burgdorferi-induced host antibodies could influence the number of viable $B$. burgdorferi as well as ospC gene transcription within murine hosts or within ticks $(29,30)$. We therefore measured the levels of viable B. burgdorferi and ospC gene expression by quantitative PCR in the feeding tick gut to determine whether the inhibitory effect of OspC

\section{Figure 3}

OspC $\mathrm{F}(\mathrm{ab})_{2}$ fragments interfere with the invasion of $B$. burgdorferi to the I. scapularis salivary glands. (a) Distribution of B. burgdorferi within the I. scapularis salivary glands (top row) or gut (bottom row) 72 hours after the onset of feeding. B. burgdorferi-infested nymphal ticks were fed on mice that had been treated with $\mathrm{F}(\mathrm{ab})_{2}$ fragments prepared from either normal rabbit sera (NRS-Fab) or polyclonal OspC sera (OspC-Fab). The spirochetes (arrows) were stained with a FITC-labeled goat antiB. burgdorferi (shown in green), and the nuclei of the gut epithelial cells were stained with propidium iodide (shown in red). Due to the lower abundance of spirochetes in the salivary gland than in gut, only a few spirochetes can be visualized through a single focal plane of the microscope. Images were recorded at $\times 400$ magnification and are presented as a merged images for clarity $(n=3)$. (b) Detection of B. burgdorferi mRNA within engorged I. scapularis salivary glands or gut. Nymphs treated with NRS-Fab or OspC-Fab were analyzed by RT-PCR for the detection of viable $B$. burgdorferi at 48 or 96 hours after the onset of feeding. Equal amounts of total RNA from ticks that fed on $\mathrm{F}(\mathrm{ab})_{2}$-treated mice were converted to CDNA with reverse transcriptase, subjected to PCR with flaB primers, and analyzed on a $1.5 \%$ agarose gel. I. scapularis $\beta$-actin was used as a control to confirm equal loading of total RNA isolated from infected, fed ticks. An aliquot of prepared RNA from each group was subjected to RT-PCR in the absence of reverse transcriptase to confirm the absence of genomic DNA (data not shown).

\section{Figure 2}

Binding of OspC $\mathrm{F}(\mathrm{ab})_{2}$ fragments to B. burgdorferi N40. (a) Binding of anti-OspC sera (black bars), normal rabbit sera (white bars), IgG, or $\mathrm{F}(\mathrm{ab})_{2}$ by ELISA. B. burgdorferi $\mathrm{N} 40$ lysates were immobilized onto microtiter wells $(10 \mu \mathrm{g} / \mathrm{ml})$ and probed with a 1:100 dilution of sera (OspC sera) or normal rabbit sera (NRS), or $8 \mu \mathrm{g} / \mathrm{ml}$ of purified $\mathrm{lgGs}$ (OspC IgG or NRS IgG) or $8 \mu \mathrm{g} / \mathrm{ml}$ of purified $\mathrm{F}(\mathrm{ab})_{2}$ fragments (OspC-Fab or NRS-Fab). Binding was detected using anti-rabbit $\mathrm{F}(\mathrm{ab})_{2}$ fragment-specific goat $\lg \mathrm{G}$ conjugated to horseradish peroxidase. Data represent the $\mathrm{OD}_{450}$ at 15 minutes (mean $\pm \mathrm{SEM}, n=3$; differences between values of wells treated with OspC or NRS Ab were at least $P<0.001)$. (b) OspC F (ab $)_{2}$ fragments directly bind to the surface of intact $B$. burgdorferi. Unfixed $B$. burgdorferi were immobilized onto glass slides and incubated in the presence of $F(a b)_{2}$ fragments prepared from normal rabbit sera (NRS-Fab) or anti-OspC sera (anti-OspC-Fab). Binding was detected using anti-rabbit $\mathrm{F}(\mathrm{ab})_{2}$ fragment-specific goat IgG labeled with FITC. Images were obtained using a $40 \times$ objective lens on a Zeiss LSM 510 confocal microscope $(n=3)$.

$\mathrm{F}(\mathrm{ab})_{2}$ fragments on B. burgdorferi invasion of salivary gland could be due to an effect on spirochete levels or downregulation of the ospC gene, respectively. Quantitative assessment of $B$. burgdorferi fla $B$ expression in feeding tick guts treated with OspC $\mathrm{F}(\mathrm{ab})_{2}$ or control $\mathrm{F}(\mathrm{ab})_{2}$ was contrasted with RT-PCR results of $B$. burgdorferi flaB expression (as shown in Figure $3 \mathrm{~b}$ ), which revealed no loss of spirochete viability within the gut (Figure 4a). We were also unable to detect significant differences in ospC expression, relative to flaB expression, when ticks were exposed to $\mathrm{F}(\mathrm{ab})_{2}$ fragments prepared from either OspC sera or normal rabbit sera (Figure $4 \mathrm{~b}$ ). Thus, OspC F(ab) $)_{2}$ fragments did not influence spirochete multiplication or ospC expression in feeding tick guts, further suggesting that OspC $\mathrm{F}(\mathrm{ab})_{2}$ fragments blocked B. burgdorferi invasion of salivary glands by interfering with the interaction of OspC with the I. scapularis salivary gland.

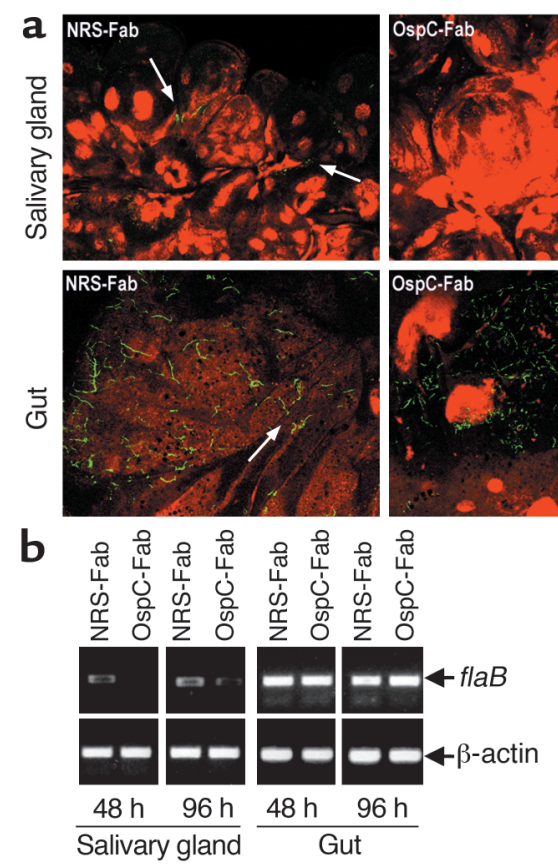




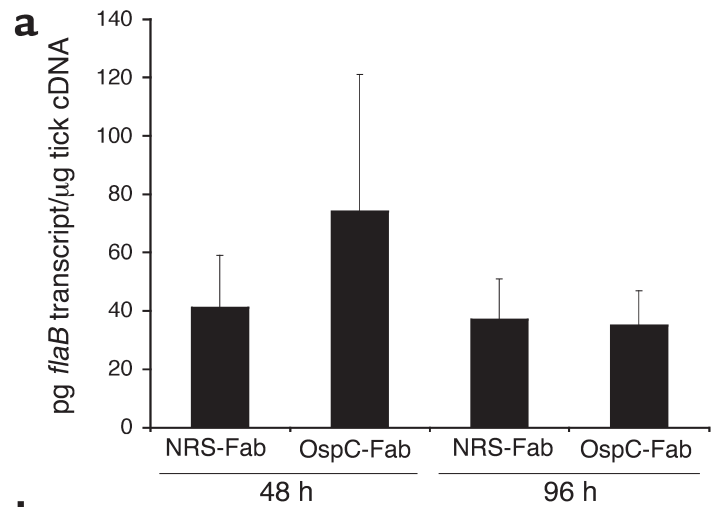

b

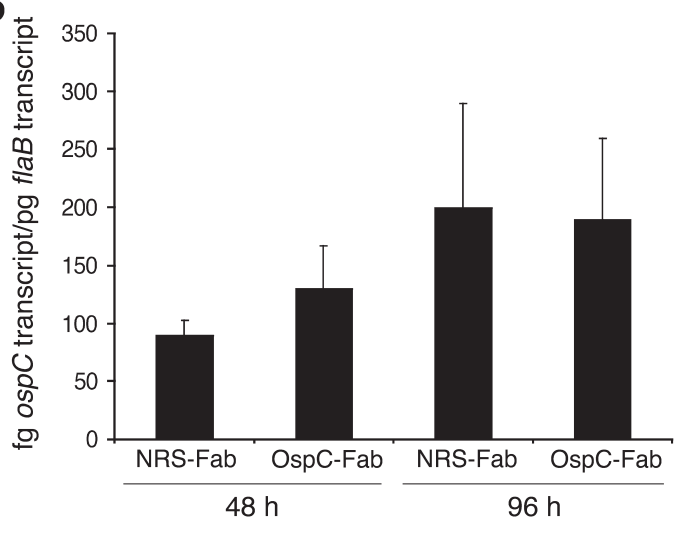

Assessment of OspC function in ticks in vivo using OspCdeficient B. burgdorferi. For further study of the role of OspC in the transmission of B. burgdorferi, an OspCdeficient mutant was generated using an infectious clone of B. burgdorferi 297. Genetic manipulation of $B$. burgdorferi is not well developed; therefore, we choose to use strain 297 instead of $\mathrm{N} 40$, as the former isolate has been successfully standardized and was used for previous genetic manipulation studies $(23,31)$. In addition, OspC proteins from N40 and 297 are highly homologous (24), both strains express ospC very efficiently, and both are highly infectious with a similar $\mathrm{ID}_{50}$ value (50-100 organisms) (U. Pal, E. Fikrig, X. Yang, and M.V. Norgard, unpublished observations; and ref. 23). The strategy for $o s p C$ inactivation is shown in Figure 5, a-c. The plasmid for transformation contained a disrupted $o s p C$ gene with an aadA marker, which confers Strep ${ }^{r}$ to wild-type B. burgdorferi. The resulting transformants with Strep ${ }^{\mathrm{r}}$ were verified by PCR for the desired addA insertion (Figure $5 \mathrm{c}$ ). None of the transformants expressed ospC mRNA or protein (Figure 6, a-c, lanes 1 and 2). To confirm that a defect associated with the OspC deficiency was solely due to the loss of ospC expression, the OspC-deficient mutant was then complemented in trans with a shuttle vector containing a wild-type ospC gene (Figure $5 \mathrm{~b}$ ). In this complemented isolate, both ospC mRNA and OspC protein were fully restored (Figure 6 , a-c, lane 3). PCR analysis of wild-type spirochetes or genetically manipulated B. burgdorferi showed similar endogenous plasmid profiles for each isolate (data not shown).

\section{Figure 4}

Measurement of viable $B$. burgdorferi and os $p C$ gene expression in feeding ticks exposed to OspC $\mathrm{F}(\mathrm{ab})_{2}$. (a) Assessment of viable $B$. burgdorferi as measured by quantitative PCR of flaB mRNA in feeding tick gut. B. burgdorferi-infected nymphs were treated with NRS-F $(a b)_{2}$ or OspC-F(ab) $)_{2}$ (NRS-Fab or OspC-Fab, respectively) were analyzed at 48 or 96 hours after the onset of feeding. Equal amounts of cDNA, converted from total RNA from each group of ticks, were subjected to quantitative PCR. Known quantities of $B$. burgdorferi DNA and $\mathrm{PCR}$ 2.1 plasmid carrying the tick $\beta$-actin gene were used to prepare standard PCR curves. Amounts of tick $\beta$-actin were determined in each samples and use to normalize the quantities of spirochete DNA between the samples. Differences in flaB RNA amounts between NRS-Fab and OspC-Fab groups of tick at 48 or 96 hours were not significant $(n=3)$. (b) Measurement of ospC transcripts in feeding tick gut by quantitative PCR as described above. NRS-Fab and OspCFab nymphs were analyzed at 48 or 96 hours after the onset of the feeding. The amounts of fla $B$ and $o s p C$ transcripts were measured in each samples and data shown are the quantities of ospC cDNA relative to flaB cDNA in normalized tick samples. Differences in ospC cDNA amounts between NRS-Fab and OspC-Fab groups of tick at 48 or 96 hours were not significant $(n=3)$.

For the generation of nymphal I. scapularis infected with wild-type or mutant $B$. burgdorferi, a microinjection method was developed to introduce spirochetes into the guts of the unfed ticks. This method ensures the loading of equal numbers of spirochetes into the tick gut and eliminates the need to produce infected nymphs from a larval feeding on B. burgdorferi-infected mice. In addition, the possibility that OspC deficiency could alter spirochete infection of mice used as blood meal host is excluded. The spirochete-injected ticks were allowed to rest within a humid chamber and were dissected at 24-hour intervals to assess the gut, salivary glands, or hemocoelic contents for the

\section{Table 2}

Effect of OspC $F(a b)_{2}$ on the invasion of B. burgdorferi to the tick salivary gland

\begin{tabular}{|c|c|c|}
\hline \multirow[t]{3}{*}{ Time } & \multicolumn{2}{|c|}{ Antibodies transferred } \\
\hline & OspC F $(a b)_{2}$ & NRS F $(a b)_{2}$ \\
\hline & \multicolumn{2}{|c|}{$\begin{array}{l}\text { (No. of spirochetes detected/ } \\
\text { tick salivary gland lobe) }\end{array}$} \\
\hline 24 hours & 0 & 0 \\
\hline 48 hours & $0.05 \pm 0.02^{\mathrm{A}}$ & $1.9 \pm 0.17$ \\
\hline 72 hours & $0.14 \pm 0.03^{B}$ & $1.7 \pm 0.11$ \\
\hline 96 hours & $0.13 \pm 0.08^{C}$ & $2.1 \pm 0.38$ \\
\hline
\end{tabular}

Naive NCr SCID mice were fed upon by B. burgdorferi-infested I. scapularis nymphs before administration of $\mathrm{F}(\mathrm{ab})_{2}$ fragments at 24 hours before tick placement. Engorged ticks were examined using confocal microscopy to enumerate B. burgdorferi within the tick salivary gland tissue from 24 to 96 hours after attachment to mice. Only a few spirochetes can be visualized through a single focal plane of the microscope; therefore, both salivary glands from each nymph were examined by scanning of the entire organ from end to end on the horizontal and vertical planes at each point. Counts were made from at least five fed ticks from each group per experiment. Each I. scapularis salivary gland contains several lobes and the average number of lobes in a salivary gland was 32 (range, 20-47). Data are the average (mean \pm SEM) number of spirochetes per lobe, counted from at least 15 ticks in each group from a total of three experiments. Differences between values in the NRS F $(a b)_{2}$-treated group are ${ }^{A} P<0.01 ;{ }^{B} P<0.01 ;$ and ${ }^{C} P<0.04$. 


\section{Table 3}

Detection of wild-type spirochetes, OspC-deficient B. burgdorferi, OspC-deficient $B$. burgdorferi complemented with a plasmid encoding OspC within the tick salivary glands

$\begin{array}{lccc}\text { Time } & \begin{array}{c}\text { Wild-type } \\ \text { (No. of spirochetes detected/tick salivary gland lobe) }\end{array} \\ \text { 24 hours } & 0.63 \pm 0.08 & 0 & 0.50 \pm 0.15 \\ 48 \text { hours } & 0.77 \pm 0.07 & 0 & 0.78 \pm 0.16 \\ 72 \text { hours } & 0.99 \pm 0.18 & 0.09 \pm 0.08 & 0.61 \pm 0.17\end{array}$

Groups of three $\mathrm{C} 3 \mathrm{H} / \mathrm{HeN}$ mice were fed upon by B. burgdorferi-infected I. scapularis nymphs ( 25 nymphs/mouse) 3 days after microinjection of spirochetes. Engorged ticks were examined using confocal microscopy to enumerate $B$. burgdorferi within the tick salivary gland tissue from 24 to 72 hours after attachment to mice. Both salivary glands from each fed nymph were examined by scanning of the entire organ from end to end on the horizontal and vertical planes at each point, and counts were made from at least five fed ticks from each group. The mean $( \pm$ SEM) number of spirochetes per salivary gland lobe from three independent experiments is presented. ADifference between OspC-deficient groups with wild-type or OspC-complemented groups at 24 hours, 48 hours, or 72 hours is at least $P<0.01(n=3)$. Wild-type, wild-type spirochetes; OspC-deficient, OspC-deficient B. burgdorferi; OspC-complemented, OspC-deficient $B$. burgdorferi complemented with a plasmid encoding OspC.

presence of viable spirochetes for a duration of 1 week. In each analysis, we found comparable numbers of live B. burgdorferi in dissected gut contents from different groups of ticks by dark-field microscopy (data not shown) and by RT-PCR (Figure 7a, left panel). All samples that were positive for RT-PCR had viable $B$. burgdorferi upon culture in BSK-H medium. We failed to detect spirochetes in dissected salivary glands or hemocoelic fluid of microinjected ticks.

We next examined the capacity of OspC-deficient spirochetes to invade tick salivary glands. Ticks were microinjected with parental or mutant spirochetes. These ticks were then allowed to engorge on mice, and the dissected salivary glands were assessed for B. burgdorferi. When guts of ticks were assessed by RT-PCR or were stained for B. burgdorferi during tick feeding, comparable numbers of spirochetes were noted within tick guts injected with wild-type spirochetes, OspC-deficient $B$. burgdorferi, or OspC-deficient B. burgdorferi complemented with OspC (Figure 7, a and b). These data indicate that wild-type spirochetes and mutant B. burgdor feri were able to survive and multiply in feeding ticks. We then assessed invasion of salivary glands by spirochetes using confocal microscopy. At 24-72 hours of feeding, when salivary glands were invaded by wild-type $B$. burgdorferi, OspC-deficient B. burgdorferi were not detected within the salivary glands (Table 3 and Figure 7c). Invasion of salivary glands was completely restored in OspC-deficient B. burgdorferi that was complemented with a plasmid encoding OspC (Table 3 and Figure 7c). We finally assessed spirochete transmission to the murine host by comparing the B. burgdorferi burden in mice that had been fed upon by ticks harboring the experimental or control spirochetes. Skin samples were collected from mice 1 week following the tick engorgement to assess the levels of OspC-deficient, OspC-com-

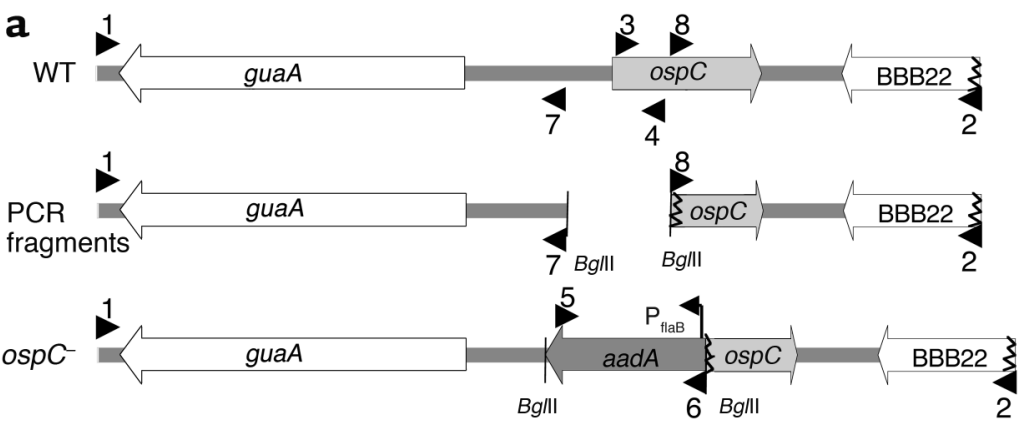

b

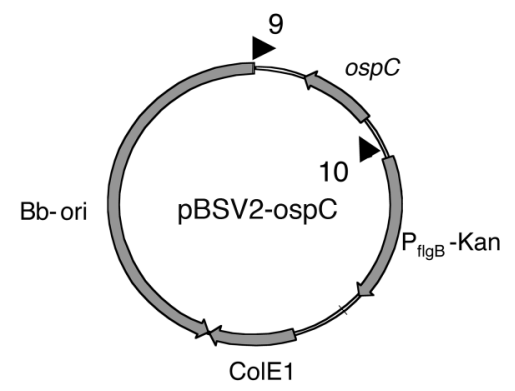

C

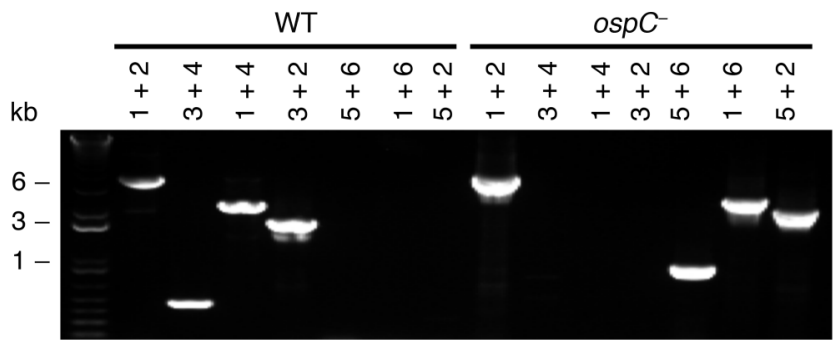

Figure 5

Inactivation and complementation of the ospC gene in B. burgdorferi. (a) Strategy for inactivation of the ospC gene (see Methods for details). Arrowheads denote numbers and positions of oligonucleotide primers for PCR analysis of mutants (osp $\left.C^{-}\right)$. guaA, GMP synthetase; BBB22, conserved hypothetical protein. (b) Diagram of the shuttle vector used for complementing the ospC mutant. Bb-ori, B. burgdorferi plasmid origin of replication; Kan, kanamycin; ColE1, E. coli plasmid origin of replication; $P$, promoter. (c) PCR analysis of the OspC-deficient $B$. burgdorferi. Number combinations above lanes indicate the primer pairs from a used for the PCR. Lane at the left is a DNA ladder; left margin, molecular sizes. 


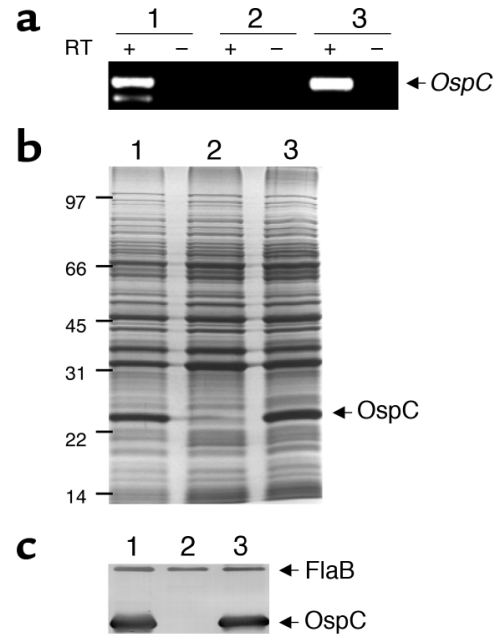

plemented, and wild-type B. burgdorferi by quantitative PCR using flaB primers. Comparable levels of wild-type spirochetes $(9.6 \pm 1.2 \mathrm{fg} f \mathrm{flaB} / \mu \mathrm{g}$ total DNA) as well as OspC-complemented B. burgdorferi $(8.0 \pm 2.4 \mathrm{fg} f \mathrm{laB} / \mu \mathrm{g}$ total DNA) were detected within the murine skin samples at 12 days following tick engorgement. In contrast, much lower levels of OspC-deficient B. burgdorferi $(0.01 \pm 0.003 \mathrm{fg} f l a B / \mu \mathrm{g}$ total DNA were detected within murine skin samples (differences with the levels of wild-type or complemented isolates were highly significant, at least $P<0.008$ ). These data indicate that OspC-

\section{Figure 7}

OspC-deficient B. burgdorferi persists within the I. scapularis gut but fails to invade the salivary gland during feeding. (a) Detection of $B$. burgdorferi mRNA within guts of unfed or engorged I. scapularis which were microinjected with wild-type spirochetes (lane 1), OspC-deficient B. burgdorferi (lane 2), or OspC-deficient spirochetes that were complemented with a plasmid expressing ospC (lane 3). Injected unfed nymphs were analyzed by RT-PCR for the detection of viable B. burgdorferi at 7 days after injection or 24-72 hours after the onset of feeding. Equal amounts of total RNA from ticks ( 25 ticks/group) were converted to $C D N A$ with reverse transcriptase, subjected to PCR with flaB primers, and analyzed on a $1.5 \%$ agarose gel. I. scapularis $\beta$-actin was used as a control to confirm equal loading of total RNA isolated from infected ticks. An aliquot of prepared RNA from each group was subjected to RT-PCR in the absence of reverse transcriptase to confirm the absence of genomic DNA (data not shown). (b) Distribution of microinjected wild-type B. burgdorferi (WT), OspCdeficient B. burgdorferi (OspC-deficient), and OspC-deficient $B$. burgdorferi complemented with a plasmid carrying the osp $C$ gene (OspC-complemented) within I. scapularis gut at 24 or 72 hours of feeding. The spirochetes (arrows) were stained with FITC-labeled goat anti-B. burgdorferi (shown in green), and the nuclei of the gut epithelial cells were stained with propidium iodide (shown in red). Images were obtained at $\times 400$ magnification and are presented as merged images for clarity. (c) Migration of microinjected spirochetes from feeding gut, as shown in b, to salivary glands at 24-72 hours of feeding. Salivary glands were invaded by wild-type and OspC-complemented B. burgdorferi (arrows); no spirochetes were detected within the salivary glands of ticks that were microinjected with OspC-deficient $B$. burgdorferi. Images were obtained at $\times 400$ magnification and are presented as merged images for clarity $(n=3)$.

\section{Figure 6}

Characterization of OspC-deficient B. burgdorferi. (a-c) Lane 1, wildtype B. burgdorferi; lane 2, OspC-deficient B. burgdorferi; lane 3, OspCdeficient $B$. burgdorferi complemented with the shuttle vector harboring a wild-type ospC gene. (a) RT-PCR analysis of ospC expression in $B$. burgdorferi isolates. The presence $\left(^{+}\right)$or absence $(-)$of reverse transcriptase (RT) in RT-PCR reaction is indicated above lanes. (b) SDS-PAGE (Coomassie blue stain) analyses of the B. burgdorferi isolates. Numbers along the left margin indicate positions of protein molecular mass markers in kDa. (c) Western blot analysis of samples corresponding to the gel shown in b. B. burgdorferi FlaB or OspC antibodies were used to detect the respective proteins (arrows).

deficient $B$. burgdorferi have a markedly reduced capacity (approximately 800-fold less than control spirochetes) for successful transmission to mice.

\section{Discussion}

B. burgdorferi is an obligate parasite of both ticks and mammals (1,3). Differential display of surface lipoproteins by $B$. burgdorferi plays a central role in spirochete adaptation to these phylogenetically diverse environments. In the vector-host transmission cycle, the spirochetes alter their surface structure inside the engorging tick $(16,32)$. B. burgdorferi upregulates ospA during entry into ticks, and this protein contributes to the colonization of spirochetes within the vector gut $(12,20)$. B. burgdorferi, in contrast, does not produce abundant OspC in the gut of an unfed tick (11). When a tick feeds

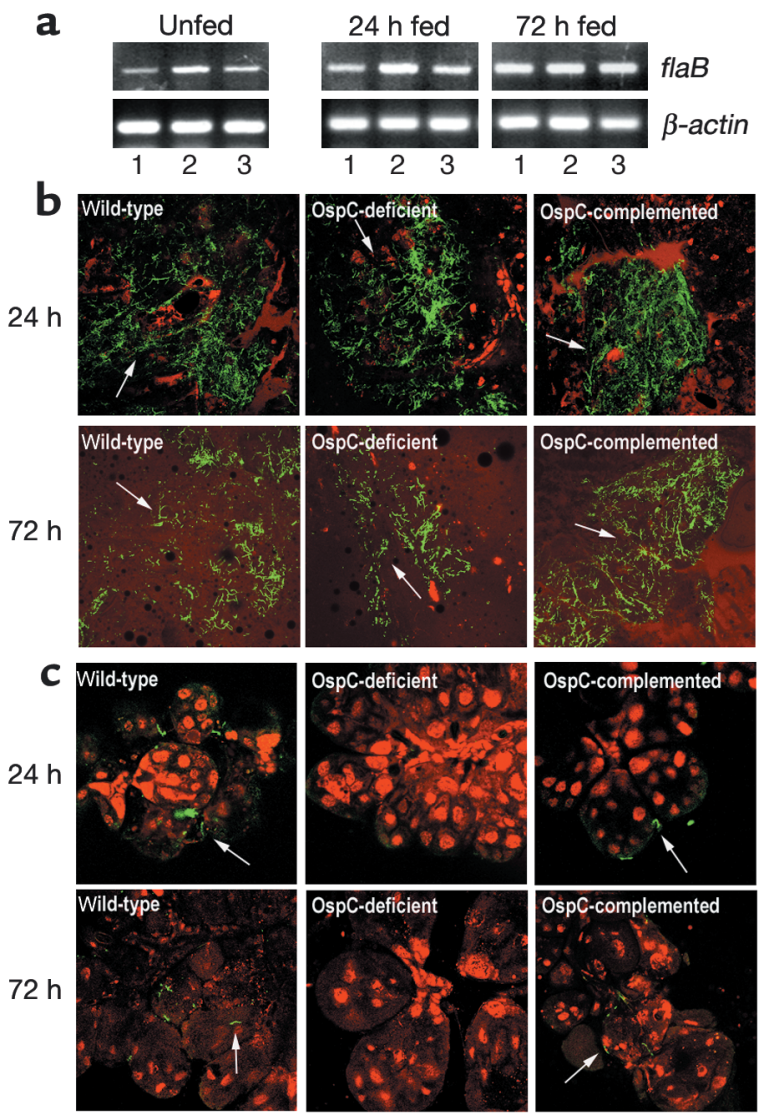


on a mammal, B. burgdorferi within the gut swiftly upregulates ospC and invades the salivary gland, and is then transmitted to the host dermis along with tick saliva (14). Although the feeding tick gut may harbor a mixed population of spirochetes with variable ospC expression, $B$. burgdorferi found within the tick salivary glands usually produce $\mathrm{OspC}(32,33)$. Spirochetes also generally continue to express osp $C$ in the reservoir host (34). This protein, therefore, has been proposed to have functions in transmission from the vector (17) and in early mammalian infection $(16,18,35)$. Here, we demonstrate that OspC binds strongly with I. scapularis salivary gland and that $\mathrm{OspC} \mathrm{F}(\mathrm{ab})_{2}$ fragments interfere with spirochete invasion of the salivary gland during tick feeding. Targeted ablation of OspC in infectious $B$. burgdorferi also prevented salivary gland invasion of spirochetes in feeding ticks, which was completely restored when OspC-deficient B. burgdorferi were complemented with a plasmid encoding OspC.

The $\mathrm{F}(\mathrm{ab})_{2}$-blocking study provides initial insight into the function of $\mathrm{OspC}$ in the B. burgdorferi life cycle. Although OspC is polymorphic in isolates of B. burgdorferi, strain-specific bactericidal activity of OspC antibodies have been reported (36). One earlier study suggested that active immunization with OspC prevented spirochete transmission (17). However, it is possible that this was due to killing of the organism in the feeding tick gut rather than specific interference with the function of $B$. burgdorferi OspC (18). To minimize the bactericidal activity of OspC antibodies in the feeding tick gut, we generated OspC $\mathrm{F}(\mathrm{ab})_{2}$ fragments and assessed binding of $\mathrm{F}(\mathrm{ab})_{2}$ fragments to intact unfixed spirochetes. Our in vivo studies demonstrated that $\mathrm{OspC} \mathrm{F}(\mathrm{ab})_{2}$ fragments, but not control $\mathrm{F}(\mathrm{ab})_{2}$ fragments, significantly prevented $B$. burgdorferi from invading the I. scapularis salivary gland. The small proportion of B. burgdorferi invaded the salivary glands in the presence of OspC F(ab) $)_{2}$ fragments (Table 2 ) is consistent with a previous study that suggested that a minor population of spirochetes, which do not produce $\mathrm{OspC}$, can be detected at the tick bite site of the murine dermis; however, the authors of that study were unable to establish infection in mice (32). The inhibition of spirochete invasion of tick salivary glands by $\mathrm{OspC} \mathrm{F}(\mathrm{ab})_{2}$ fragments was due to direct interference with OspC function rather than to any residual bactericidal activity, as quantitative RT-PCR demonstrated comparable numbers of viable spirochetes within the guts of feeding ticks exposed to control or OspC F(ab $)_{2}$ fragments. OspC $\mathrm{F}(\mathrm{ab})_{2}$ fragments prepared from the polyclonal antisera are therefore likely to bind epitope(s) on OspC and interfere with the B. burgdorferi adherence to the tick salivary gland.

It has been suggested that expression of $B$. burgdorferi ospC may be influenced by host-generated immune responses or by passive transfer of $B$. burgdorferi antibodies to the murine host or to feeding ticks $(29,30,37)$. Passive immunization of mice with OspA antibodies altered B. burgdorferi numbers and resulted in reduced ospC expression by spirochetes in feeding ticks; it was postulated that this could be due to a quorum-sensing mech- anism (29). To prove that the inhibition of spirochete invasion of tick salivary gland by $\mathrm{OspC} \mathrm{F}(\mathrm{ab})_{2}$ fragments in our in vivo study was not associated with the downregulation of ospC expression by B. burgdorferi in the feeding gut, osp $C$ transcripts relative to fla $B$ transcripts were measured by quantitative RT-PCR. The B. burgdorferifla $B$ gene served as a control gene (22); quantities of $f l a B$ mRNA increase linearly during spirochete multiplication in feeding ticks (38). Thus, measurement of ospC transcripts in flaB-positive $B$. burgdorferi allowed us to quantitate viable OspC-producing spirochetes. $B$. burgdorferi ospC expression in ticks exposed to $\mathrm{F}(\mathrm{ab})_{2}$ fragments prepared either from normal rabbit sera or OspC antisera was similar, indicating that $\mathrm{OspC} \mathrm{F}(\mathrm{ab})_{2}$ fragments did not influence ospC expression by spirochetes.

Inhibition of spirochete salivary gland colonization by OspC $\mathrm{F}(\mathrm{ab})_{2}$ fragments suggested the involvement of OspC in this process. However, $\mathrm{F}(\mathrm{ab})_{2}$-mediated interference could also result from steric hindrance of other potential ligands on the spirochete surface. To precisely implicate OspC in salivary gland colonization, we generated an OspC-deficient B. burgdorferi and determined whether B. burgdorferi lacking OspC could significantly invade the tick salivary gland. Recent efforts toward the genetic manipulation of $B$. burgdorferi to explore protein function have had limited success $(23,31,39,40)$. The OspC-deficient $B$. burgdorferi generated in earlier studies (41) could not be used in our study of OspC function, as they were derived from noninfectious, high-passage spirochetes that expressed ospC poorly and had lost many of the endogenous plasmids. Successful generation of an OspC-deficient spirochete in a low-passage, infectious isolate of B. burgdorferi allowed us to demonstrate that $\mathrm{OspC}$ production is necessary for significant invasion of tick salivary glands by spirochetes as well as for their transmission to mice. Genetic complementation (42) of the mutant is necessary for conclusive assessment of spirochete gene function $(23,43)$. We therefore complemented the mutant with a wild-type ospC gene and showed that the inability of the OspC-deficient spirochetes to colonize salivary glands was due to the loss of OspC rather than to any other potential aberration caused by the genetic manipulation process. We developed a microinjection method to introduce spirochetes into ticks, which will be an invaluable tool for future studies investigating transmission of spirochetes from ticks to hosts using other B. burgdorferi mutants.

The molecular interaction between microbes and arthropods is important for the transmission and persistence of pathogens in a complex enzootic life cycle. Many arthropod-borne pathogens are transmitted to a host via the salivary gland during the blood meal (44). OspC, a feeding-induced B. burgdorferi ligand, participates in pathogen colonization of I. scapularis salivary glands during engorgement. It was recently shown that ospC expression is controlled by the Rrp2-RpoN-RpoS regulatory pathway in vitro $(23,45)$. It remains to be determined, however, whether this pathway governs the differential expression of os $p C$ during vector-borne trans- 
mission of B. burgdorferi. Our results underscore the utility of genetic manipulation of infectious spirochetes for characterization of ligand-receptor interactions at the arthropod-pathogen interface. Understanding the microbial and vector ligands that participate in pathogen transmission may lead to new vector-based approaches to interfere with the B. burgdorferi life cycle, thus reducing the incidence of Lyme disease.

\section{Acknowledgments}

We sincerely thank Aravinda M. de Silva and Ruth R. Montgomery for their comments and suggestion in the preparation of this manuscript. We gratefully acknowledge the help provided by Debby Beck, Syed A. Morshed, Denise Lusitani, Lilin Xiang, and Sophie M. Alani in this study. This work has been supported by the grants from the NIH. E. Fikrig is the recipient of a Burroughs Wellcome Clinical Scientist Award in Translational Research. R.A. Flavell is an Investigator of the Howard Hughes Medical Institute.

1. Nadelman, R.B., and Wormser, G.P. 1998. Lyme borreliosis. Lancet. 352:557-565.

2. Steere, A.C. 2001. Lyme disease. N. Engl. J. Med. 345:115-125.

3. de Silva, A.M., and Fikrig, E. 1997. Arthropod- and host-specific gene expression by Borrelia burgdorferi. J. Clin. Invest. 99:377-379.

4. Steere, A.C., et al. 1983. The early clinical manifestations of Lyme disease. Ann. Intern. Med. 99:76-82.

5. Barthold, S.W., deSouza, M.S., Janotka, J.L., Smith, A.L., and Persing, D.H. 1993. Chronic Lyme borreliosis in the laboratory mouse. Am.J. Pathol. 143:959-971.

6. Pal, U., and Fikrig, E. 2003. Adaptation of Borrelia burgdorferi in the vector and vertebrate host. Microbes Infect. 5:659-666.

7. Fikrig, E., et al. 1997. Borrelia burgdorferi P35 and P37 proteins, expressed in vivo, elicit protective immunity. Immunity. 6:531-539.

8. Guo, B.P., Brown, E.L., Dorward, D.W., Rosenberg, L.C., and Hook, M. 1998. Decorin-binding adhesins from Borrelia burgdorferi. Mol. Microbiol. 30:711-723.

9. Hellwage, J., et al. 2001. The complement regulator factor $\mathrm{H}$ binds to the surface protein OspE of Borrelia burgdorferi. J. Biol. Chem. 276:8427-8435.

10. Zhang, J.R., Hardham, J.M., Barbour, A.G., and Norris, S.J. 1997. Antigenic variation in Lyme disease Borreliae by promiscuous recombination of Vmp-like sequence cassettes. Cell. 89:275-285.

11. Schwan, T.G., and Piesman, J. 2000. Temporal changes in outer surface proteins $\mathrm{A}$ and $\mathrm{C}$ of the Lyme disease-associated spirochete, Borrelia burgdorferi, during the chain of infection in ticks and mice. J. Clin. Microbiol. 38:382-388.

12. Pal, U., et al. 2000. Attachment of Borrelia burgdorferi within Ixodes scapularis mediated by outer surface protein A. J. Clin. Invest. 106:561-569.

13. Marconi, R.T., Samuels, D.S., and Garon, C.F. 1993. Transcriptional analyses and mapping of the ospC gene in Lyme disease spirochetes. J. Bacteriol. 175:926-932.

14. Schwan, T.G., Piesman, J., Golde, W.T., Dolan, M.C., and Rosa, P.A. 1995. Induction of an outer surface protein on Borrelia burgdorferi during tick feeding. Proc. Natl. Acad. Sci. U. S. A. 92:2909-2913.

15. de Silva, A.M., Telford, S.R., Brunet, L.R., Barthold, S.W., and Fikrig, E. 1996. Borrelia burgdorferi OspA is an arthropod-specific transmissionblocking Lyme disease vaccine. J. Exp. Med. 183:271-275.

16. Schwan, T.G., and Piesman, J. 2002. Vector interactions and molecular adaptations of Lyme disease and relapsing fever spirochetes associated with transmission by ticks. Emerg. Infect. Dis. 8:115-121.

17. Gilmore, R.D., Jr., and Piesman, J. 2000. Inhibition of Borrelia burgdorferi migration from the midgut to the salivary glands following feeding by ticks on OspC-immunized mice. Infect. Immun. 68:411-414.

18. Schwan, T.G. 2003. Temporal regulation of outer surface proteins of the Lyme-disease spirochaete Borrelia burgdorferi. Biochem. Soc. Trans. 31:108-112.

19. Bockenstedt, L.K., et al. 1997. Borrelia burgdorferi strain-specific Osp Cmediated immunity in mice. Infect. Immun. 65:4661-4667.

20. Pal, U., et al. 2001. Inhibition of Borrelia burgdorferi-tick interactions in vivo by outer surface protein A antibody. J. Immunol. 166:7398-7403.
21. Li, S., Kwon, J., and Aksoy, S. 2001. Characterization of genes expressed in the salivary glands of the tsetse fly, Glossina morsitans morsitans. Insect Mol. Biol. 10:69-76.

22. Anguita, J., et al. 2000. Borrelia burgdorferi gene expression in vivo and spirochete pathogenicity. Infect. Immun. 68:1222-1230.

23. Hubner, A., et al. 2001. Expression of Borrelia burgdorferi OspC and DbpA is controlled by a RpoN-RpoS regulatory pathway. Proc. Natl. Acad. Sci. U. S. A. 98:12724-12729.

24. Fung, B.P., McHugh, G.L., Leong, J.M., and Steere, A.C. 1994. Humoral immune response to outer surface protein $\mathrm{C}$ of Borrelia burgdorferi in Lyme disease: role of the immunoglobulin $\mathrm{M}$ response in the serodiagnosis of early infection. Infect. Immun. 62:3213-3221.

25. Bono, J.L., et al. 2000. Efficient targeted mutagenesis in Borrelia burgdorferi. J. Bacteriol. 182:2445-2452.

26. Frank, K.L., Bundle, S.F., Kresge, M.E., Eggers, C.E., and Samuels, D.S. 2003. aadA confers streptomycin resistance in Borrelia burgdorferi. J. Bacteriol. 185:6723-6727.

27. Eggers, C.H., et al. 2002. Identification of loci critical for replication and compatibility of a Borrelia burgdorferi cp32 plasmid and use of a cp32based shuttle vector for the expression of fluorescent reporters in the Lyme disease spirochaete. Mol. Microbiol. 43:281-295.

28. Stewart, P., Thalken, R., Bono, J., and Rosa, P. 2001. Isolation of a circular plasmid region sufficient for autonomous replication and transformation of infectious Borrelia burgdorferi. Mol. Microbiol. 39:714-722.

29. de Silva, A.M., et al. 1999. Influence of outer surface protein A antibody on Borrelia burgdorferi within feeding ticks. Infect. Immun. 67:30-35.

30. Liang, F.T., Jacobs, M.B., Bowers, L.C., and Philipp, M.T. 2002. An immune evasion mechanism for spirochetal persistence in Lyme borreliosis. J. Exp. Med. 195:415-422.

31. Hubner, A., Revel, A.T., Nolen, D.M., Hagman, K.E., and Norgard, M.V. 2003. Expression of a luxS gene is not required for Borrelia burgdorferi infection of mice via needle inoculation. Infect. Immun. 71:2892-2896.

32. Ohnishi, J., Piesman, J., and de Silva, A.M. 2001. Antigenic and genetic heterogeneity of Borrelia burgdorferi populations transmitted by ticks. Proc. Natl. Acad. Sci. U. S. A. 98:670-675.

33. Fingerle, V., et al. 2002. Dynamics of dissemination and outer surface protein expression of different European Borrelia burgdorferi sensu lato strains in artificially infected Ixodes ricinus nymphs. J. Clin. Microbiol. 40:1456-1463.

34. Montgomery, R.R., Malawista, S.E., Feen, K.J., and Bockenstedt, L.K. 1996. Direct demonstration of antigenic substitution of Borrelia burgdorferi ex vivo: exploration of the paradox of the early immune response to outer surface proteins A and C in Lyme disease. J. Exp. Med. 183:261-269.

35. Pohl-Koppe, A., Kaunicnik, A., and Wilske, B. 2001. Characterization of the cellular and humoral immune response to outer surface protein $\mathrm{C}$ and outer surface protein 17 in children with early disseminated Lyme borreliosis. Med. Microbiol. Immunol. (Berl) 189:193-200.

36. Hovius, J.W., Hovius, K.E., Oei, A., Houwers, D.J., and van Dam, A.P. 2000. Antibodies against specific proteins of and immobilizing activity against three strains of Borrelia burgdorferi sensu lato can be found in symptomatic but not in infected asymptomatic dogs. J. Clin. Microbiol. 38:2611-2621.

37. Liang, F.T., Nelson, F.K., and Fikrig, E. 2002. Molecular adaptation of Borrelia burgdorferi in the murine host. J. Exp. Med. 196:275-280.

38. Hodzic, E., Feng, S., Freet, K.J., Borjesson, D.L., and Barthold, S.W. 2002. Borrelia burgdorferi population kinetics and selected gene expression at the host-vector interface. Infect. Immun. 70:3382-3388.

39. Purser, J.E., et al. 2003. A plasmid-encoded nicotinamidase (PncA) is essential for infectivity of Borrelia burgdorferi in a mammalian host. Mol. Microbiol. 48:753-764.

40. Coburn, J., and Cugini, C. 2003. Targeted mutation of the outer membrane protein P66 disrupts attachment of the Lyme disease agent, Borrelia burgdorferi, to integrin $\alpha_{v} \beta_{3}$. Proc. Natl. Acad. Sci. U. S. A. 100:7301-7306.

41. Tilly, K., et al. 1997. The Borrelia burgdorferi circular plasmid cp26: conservation of plasmid structure and targeted inactivation of the ospC gene. Mol. Microbiol. 25:361-373.

42. Elias, A.F., Schmutzhard, J., Stewart, P.E., Schwan, T.G., and Rosa, P. 2002. Population dynamics of a heterogeneous Borrelia burgdorferi B31 strain in an experimental mouse-tick infectious cycle. Wien. Klin. Wochenschr. 114:557-561.

43. Sartakova, M.L., et al. 2001. Complementation of a nonmotile flaB mutant of Borrelia burgdorferi by chromosomal integration of a plasmid containing a wild-type flaB allele. J. Bacteriol. 183:6558-6564.

44. Munderloh, U.G., and Kurtti, T.J. 1995. Cellular and molecular interrelationships between ticks and prokaryotic tick-borne pathogens. Annu. Rev. Entomol. 40:221-243.

45. Yang, X.F., Alani, S.M., and Norgard, M.V. 2003. The response regulator Rrp2 is essential for the expression of major membrane lipoproteins in Borrelia burgdorferi. Proc. Natl. Acad. Sci. U. S. A. 100:11001-11006. 